\title{
Rhenium Complex with 2-[(2-Pyridylmethyl)amino]ethanethiol
}

\author{
Masahiro Mikuriya, ${ }^{\dagger}$ Tetsuji Watanabe, Aiko Suyama, and Daisuke Yoshioka \\ Department of Chemistry and Research Center for Coordination Molecule-based Devices, School of Science \\ and Technology, Kwansei Gakuin University, 2-1 Gakuen, Sanda 669-1337, Japan
}

\begin{abstract}
A rhenium(VII) complex with 2-[(2-pyridylmethyl)amino]ethanethiol (Hpmaet), [Re(pmaet) $\left.\mathrm{O}_{3}\right]$, was synthesized. The crystal structure was determined by the single-crystal X-ray diffraction method at $123 \mathrm{~K}$. It crystallizes in the monoclinic space group $P 2{ }_{1} / n$ with $a=10.054(5) \AA, b=8.466(4) \AA, c=12.924(7) \AA, \beta=108.552(9)^{\circ}, V=1043.0(10) \AA^{3}, D_{\mathrm{x}}=2.557 \mathrm{~g} /$ $\mathrm{cm}^{3}$, and $Z=4$. The $R 1[I>2 \sigma(I)]$ and $w R 2$ (all data) values are 0.0195 and 0.0545 , respectively, for all 2401 independent reflections. The compound consists of an octahedral molecule of rhenium(VII) with facial pmaet and three oxido oxygens.
\end{abstract}

(Received October 22, 2014; Accepted November 29, 2014; Published on web January 10, 2015)

There has been considerable interest in the coordination chemistry of metal complexes with organic thiolic ligands because of their various structures involving oligonuclear and polynuclear metal atoms. ${ }^{1-10} \quad$ 2-[(2-Pyridylmethyl)amino]ethanethiol (Hpmaet) is an interesting thiolic ligand to make such compounds, giving a trinuclear zinc(II) complex, $\left[\mathrm{Zn}\left\{\mathrm{Zn}(\text { pmaet })_{2}\right\}_{2}\right]\left(\mathrm{ClO}_{4}\right)_{2},{ }^{5}$ a trinuclear cadmium(II) complex, $\left[\mathrm{Cd}\left\{\mathrm{Cd}(\text { pmaet })_{2}\right\}_{2}\right]\left(\mathrm{ClO}_{4}\right)_{2},{ }^{8}$ a tetranuclear palladium(II) complex, $\quad\left[\mathrm{Pd}_{4}(\text { pmaet })_{4}\right]\left(\mathrm{ClO}_{4}\right)_{3} \mathrm{Cl},{ }^{7}$ and a polynucler manganese(II) complex, $[\mathrm{Mn}(\mathrm{pmaet}) \mathrm{Cl}]_{n} \cdot{ }^{3}$ However, there has been no report on a rhenium complex with Hpmaet. In this study, we synthesized a new complex from a reaction of ammonium perrhenate(VII) and Hpmaet, and determined the crystal structure of this compound, which shows an octahedral rhenium(VII) with three donor atoms of pmaet and three oxidooxygen atoms, as shown in Fig. 1.

The Hpmaet ligand was synthesized by a reaction of 2-aminomethylpyridine with ethylene sulfide according to a literature method. ${ }^{2}$ To a solution of Hpmaet $(75 \mathrm{mg}, 0.44$ $\mathrm{mmol}$ ) in $4 \mathrm{~cm}^{3}$ of methanol was added two drops of triethylamine $(24 \mathrm{mg}, 0.24 \mathrm{mmol})$. A solution of ammonium perrhenate $(103 \mathrm{mg}, 0.38 \mathrm{mmol})$ in $4 \mathrm{~cm}^{3}$ of methanol was added with stirring dropwise under argon. The resulting solution was left several days to give colorless crystals. The

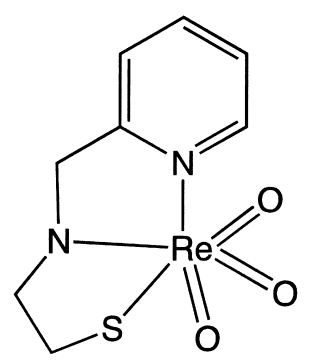

Fig. 1 Chemical structure of $\left[\mathrm{Re}(\mathrm{pmaet}) \mathrm{O}_{3}\right]$.

$\dagger$ To whom correspondence should be addressed.

E-mail: junpei@kwansei.ac.jp complexes were collected. X-ray diffraction data for these crystals were collected at $123 \mathrm{~K}$ on a Bruker CCD X-ray diffractometer (SMART APEX) using graphite-monochromated Mo- $K_{\alpha}$ radiation. Crystal data and details concerning data collection are given in Table 1 . The structure was solved by direct methods and refined by full-matrix least-squares methods. The hydrogen atoms were included using the riding atom model. All of the calculations were carried out on a Pentium IV Windows 2000 computer utilizing the SHELXTL software package. Crystallographic data have been deposited with Cambridge Crystallographic Data Centre: Deposit number

Table 1 Crystal and experimental data

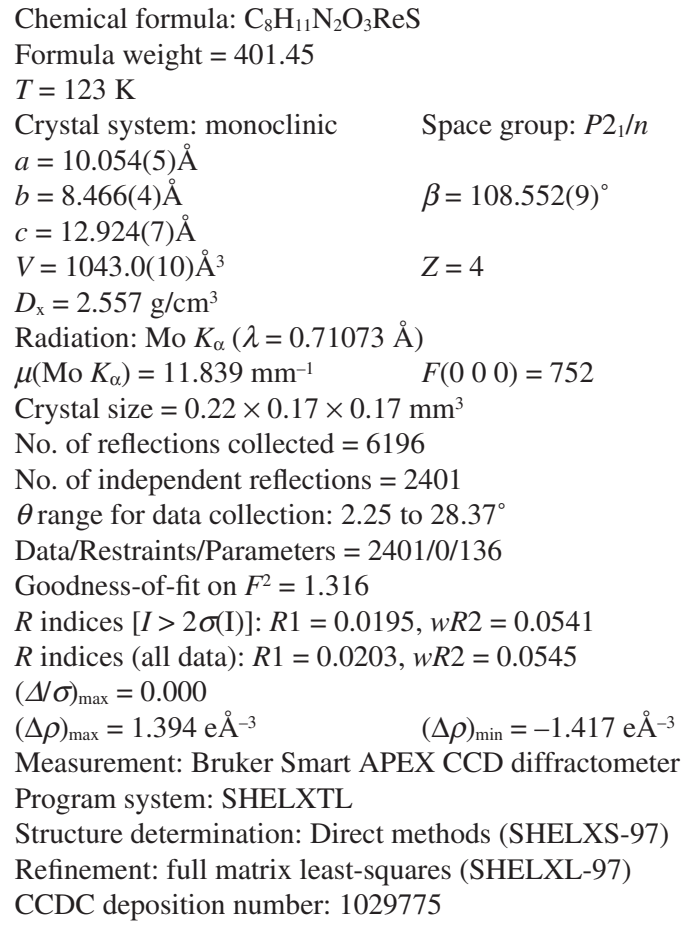




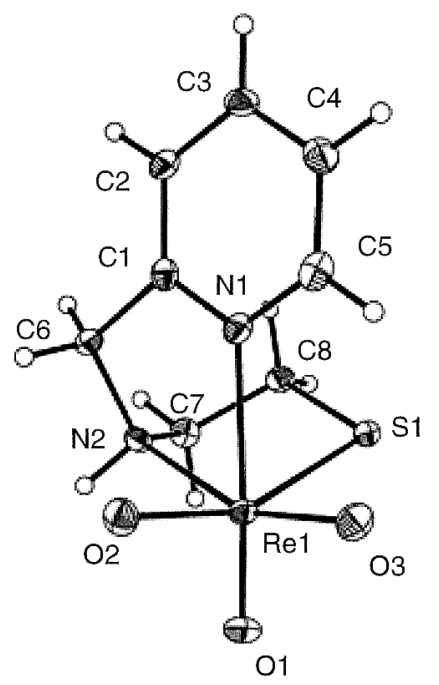

Fig. 2 ORTEP view of $\left[\operatorname{Re}(\right.$ pmaet $\left.) \mathrm{O}_{3}\right]$. The thermal ellipsoids are shown at the $50 \%$ probability level.

Table 2 Selected bond distances $(\AA)$ and angles $\left({ }^{\circ}\right)$

\begin{tabular}{lclc}
\hline Re1-S1 & $2.5052(12)$ & Re1-N1 & $2.277(3)$ \\
Re1-N2 & $2.245(3)$ & Re1-O1 & $1.726(3)$ \\
Re1-O2 & $1.729(2)$ & Rel-O3 & $1.730(2)$ \\
S1-Re1-N1 & $75.83(8)$ & S1-Re1-N2 & $78.15(7)$ \\
S1-Re1-O1 & $91.95(9)$ & S1-Re1-O2 & $155.00(8)$ \\
S1-Re1-O3 & $87.54(9)$ & N1-Re1-N2 & $73.26(10)$ \\
N1-Re1-O1 & $161.70(12)$ & N1-Re1-O2 & $83.91(10)$ \\
N1-Re1-O3 & $87.13(10)$ & N2-Re1-O1 & $91.10(11)$ \\
N2-Re1-O2 & $82.12(10)$ & N2-Re1-O3 & $157.97(10)$ \\
O1-Rel-O2 & $103.71(11)$ & O1-Re1-O3 & $106.21(12)$ \\
O2-Re1-O3 & $106.14(11)$ & & \\
\hline
\end{tabular}

CCDC-1029775. Copies of the data can be obtained free of charge via http://www.ccdc.cam.ac.uk/conts/retrieving.html (or from the Cambridge Crystallographic Data Centre, 12, Union Road, Cambridge, CB2 1EZ, UK; Fax: +44 1223 336033; e-mail: deposit@ccdc.cam.ac.uk).

The molecular structures drawn by ORTEP are shown in Fig. 2. Selected bond distances and angles are given in Table 2. The asymmetric unit consists of one $\left[\operatorname{Re}(\right.$ pmaet $\left.) \mathrm{O}_{3}\right]$ molecule. In this molecule, the rhenium atom is coordinated by the thiolatosulfur S1, amino-nitrogen N2, and pyridyl-nitrogen N1 atoms of the pmaet ligand and three oxido-oxygen $\mathrm{O} 1, \mathrm{O} 2$, and $\mathrm{O} 3$ atoms in a distorted octahedron. It is to be noted that the pmaet ligand is coordinated to the metal atom in a facial mode. The Re1-S1 bond length is $2.5052(12) \AA$, which is significantly longer than those found in related thiolato rhenium complexes. ${ }^{10}$ The Re1N2 distance $(2.245(3) \AA)$ is significantly shorter than the Re1N1 distance $(2.277(3) \AA)$, showing that the amino nitrogen atom is bonded to the central atom more strongly. The Re1-N2-C7C8-S1 chelate ring has a gauche conformation, and C7 and C8 atoms are located $0.614 \AA$ above and $-0.032 \AA$ below the plane defined by Re1-N2-S1, respectively. On the other hand, the

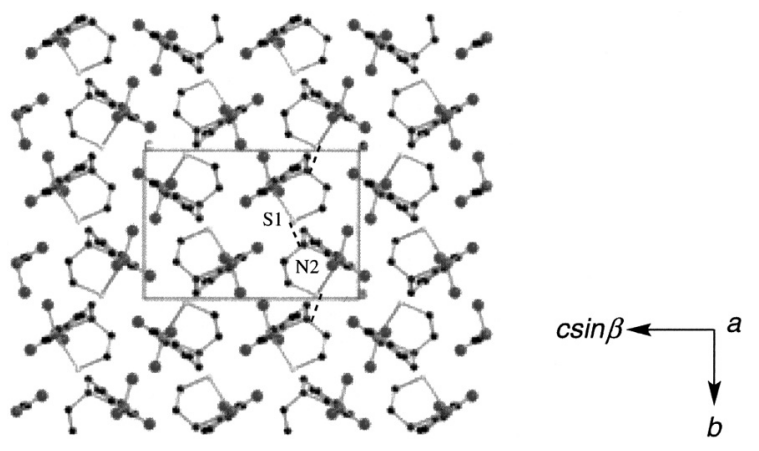

Fig. 3 Packing diagram of $\left[\mathrm{Re}\right.$ (pmaet) $\left.\mathrm{O}_{3}\right]$ viewed along the $a$ axis.

Re1-N1-C1-C6-N2 chelate ring has an envelope conformation, and $\mathrm{C} 1$ and $\mathrm{C} 6$ are both located $0.201 \AA$ and $0.617 \AA$ above the Re1-N1-N2 plane, respectively. In the crystal, the amino group of the pmaet ligand of the complex forms hydrogen bondings with the thiolato-sulfur atom of the neighboring complex molecule [N2-H‥S $1(-x+3 / 2, y-1 / 2,-z+1 / 2) 3.246(3) \AA]$ to form a polymeric chain along the $b$ axis (Fig. 3).

\section{Acknowledgements}

The present work was partially supported by Grant-in-Aid for Scientific Research No. 26410080 from the Ministry of Education, Culture, Sports, Science and Technology (MEXT, Japan) and the NEXT-Supported Program for the Strategic Research Foundation at Private Universities, 2010 - 2014.

\section{References}

1. B. Krebs and G. Henkel, Angew. Chem. Int. Ed. Engl., 1991, 30, 769.

2. M. Handa, M. Mikuriya, Z. J. Zhong, H. Okawa, and S. Kida, Bull. Chem. Soc. Jpn., 1988, 61, 3883.

3. M. Mikuriya, F. Adachi, H. Iwasawa, M. Handa, M. Koikawa, and H. Okawa, Bull. Chem. Soc. Jpn., 1994, 67, 3263.

4. M. Mikuriya, T. Kotera, F. Adachi, M. Handa, M. Koikawa, and H. Okawa, Bull. Chem. Soc. Jpn., 1995, 68, 574.

5. M. Mikuriya, X. Jian, S. Ikemi, T. Kawahashi, and H. Tsutsumi, Bull. Chem. Soc. Jpn., 1998, 71, 2161.

6. M. Mikuriya and T. Kotera, Chem. Lett., 1998, 971.

7. T. Kawahashi, M. Mikuriya, R. Nukada, and J.-W. Lim, Bull. Chem. Soc. Jpn., 2001, 74, 323.

8. M. Mikuriya, X. Jian, S. Ikemi, T. Kawahashi, H. Tsutsumi, A. Nakasone, and J.-W. Lim, Inorg. Chim. Acta, 2001, 312, 183.

9. T. Kotera, T. Sugimoto, and M. Mikuriya, Chem. J. Moldova, 2007, 2,102.

10. S. K. Meegalla, K. Plossl, M.-P. Kung, D. A. Stevenson, M. Mu, S. Kushner, L. M. Liable-Sands, A. L. Rheingold, and H. F. Kung, J. Med. Chem., 1998, 41, 428. 